\title{
Statistical errors in medical publication
}

\section{Abstract}

While errors can be application-related, they can also occur at different stages of the study such as planning, implementation, analysis, interpretation, and presentation, all of which are related to the statistical topics. The statistical errors in the study process can be categorized as the ones that occur

(i) In the research process (before reporting)

(ii) In presentations or publications.

Some statistical errors in a publication can be assessed, while some others cannot be. In publications, not all of the statistical quantities can be checked; it is only possible to check the accuracy of some of the statistics via reported descriptive values. In addition, some terminology, demonstration and interpretation errors can be identified. As such, discussions related to the statistical errors in publications are limited to errors that can possibly be determined.

When we evaluate the statistical errors that are committed in published articles in terms of their effects on the study results, we need to acknowledge the fact that some of the errors

a) Are directly pertinent to the results, some of them,

b) Occur in demonstration and terminology only and do not affect the results.

Reviewers are expected to take part in the task of prevention of statistical errors in publications. The reviewer of a manuscript, who is an expert in the subject matter areas related to the evaluation, should request from the editor that the manuscript be reviewed by a biostatistics specialist. The most important factor in preventing statistical errors in publications is having the editor submit all manuscripts for a statistical review. Nothing can be done for errors that cannot be detected in the process of publication; nevertheless, prospectively speaking, it is important that the journal editors publish papers that carry educational qualifications on statistical topics, and are oriented towards statistical errors committed in research studies, in the sense of informing the researchers and drawing attention to the topic.

Keywords: statistical errors, statistical review, publication review
Volume 2 Issue I - 2015

\author{
Ilker Ercan,' Hakan Demirtas ${ }^{2}$ \\ 'Department of Biostatistics, Uludag University, Turkey \\ ${ }^{2}$ Division of Epidemiology and Biostatistics, University of Illinois, \\ USA
}

Correspondence: Ilker Ercan, Department of Biostatistics, Uludag University, Medical Faculty, Bursa,Turkey, Tel +90 224 295388I,Email ercan@uludag.edu.tr

Received: January 28, 2014 | Published: February 19, 2015

\section{Introduction}

With the advancement of tools for gaining knowledge, we have access to data with larger complexity and information. To understand the structures of the acquired knowledge, the data have to be analyzed, and this analysis can only be performed using statistical tools. Unfortunately, in the process of employing statistical methods in scientific research, inappropriate applications can be encountered. When statistical errors are committed, both scientists and users of scientific findings are exposed to the negative consequences. ${ }^{1}$ Mistakes and errors occur if a rule, a principle or a fact is violated in some way. When this situation occurs purposefully or unknowingly, it is defined as a "mistake" or "error", respectively. Consequently, ethical problems arise when either "errors" are committed or "mistakes" are made. Based on investigations of errors in the scientific process, the causes can be classified into the four groups:
a. Not consulting a specialist on the topic,
b. Falsely assuming that it is known,
c. Not having adequate knowledge, and
d. Carelessness.

While errors can be application-related, they can also occur at different stages of the study such as planning, implementation, analysis, interpretation, and presentation, all of which are related to the statistical topics. The statistical errors in the study process can be categorized as the ones that occur

i. In the research process (before reporting) and

ii. In presentations or publications.

Some statistical errors in a publication can be assessed, while some others cannot be. In publications, not all of the statistical quantities can be checked; it is only possible to check the accuracy of some of the statistics via reported descriptive values. In addition, some terminology, demonstration and interpretation errors can be identified. As such, discussions related to the statistical errors in publications are limited to errors that can possibly be determined. Naturally, various error classifications can be outlined based on various studies that examine the statistical errors in published articles. There is no unique definition of either "statistical error" or "statistical error rate". ${ }^{2}$ The statistical errors committed in the journals in the medical field can be summarized under the categories below ${ }^{3}$ :

A. Errors related to p-values

a. p-values given in closed form,

b. p-values lacking after statistical tests, 
c. Incorrect p-values,

d. Incorrect demonstration of p-values

B. Errors related to tests

I. Statistical technique used but not defined,

II. Insufficient data presented for the statistical test,

III. Incorrect name of the statistical test,

IV. Statistical technique defined but not used,

V. Use of incorrect test,

VI. Statistical analysis required but not performed

C. Errors in the summarization of data

D. Mathematical demonstration errors

E. Statistical symbol errors

F. Incomprehensible statistical terms

G. Inappropriate interpretation

H. Errors in (statistical) terminology

I. Incorrect and insufficient demonstration of descriptive statistics

J. Presentation of statistical method-analysis and results in the incorrect section of the manuscript

Statistical methods may be inappropriately used at every stage of medical research related to data analysis such as the design of the experiment, data collection and pre-processing, the analysis method and implementation, and interpretation. ${ }^{3}$ Using inappropriate statistical methods, techniques and analyses could be a waste of time and financial resources, and most importantly, considering scientific ethics, it is detrimental to the scientific concepts and to humanity. Even when a study is carefully planned, the use of incorrect statistical approaches may produce misleading, suboptimal, incoherent results that are amenable to being cited by other researchers. ${ }^{1}$

When we evaluate the statistical errors that are committed in published articles in terms of their effects on the study results, we need to acknowledge the fact that some of the errors

(i) Are directly pertinent to the results, some of them

(ii) Occur in demonstration and terminology only and do not affect the results.

At the publication stage, the last stage of a study, which has been reached after overcoming huge difficulties, three fundamental negative situations can ensue regarding possible negative effects of errors:

1) Publications with statistical errors induce a negative effect on science and mankind.

2) When these errors are identified during the reviewers' assessment, they will cause a loss of academic confidence in the study, leading to an early rejection.

3) Statistical errors in published articles are likely to cause a loss of an author's academic credibility.

The manuscripts submitted to journals should certainly be reviewed by biostatistics specialists, and these specialists should participate in the editor/reviewer/advisory boards of journals. Otherwise, statistical errors are more likely to be encountered in published articles. When the literature related to this topic is examined, there are various studies on the statistical errors in published articles, and most journals started requiring biostatistics specialist assessments. ${ }^{1,3,4-9}$ Editors ascribe substantial importance to studies the draw attention to this topic, and they publish this type of studies in their journals. ${ }^{1-11}$

Researchers who do not have a solid statistical background commit errors in the utilization of statistics in their studies. In some cases, although researchers obtain statistical counseling, they commit errors in the presentation while writing papers. In conclusion, it is of major importance that submitted manuscripts be reviewed in terms of statistical aspects to prevent statistical and interpretation errors. ${ }^{3}$

In some journals, following the pre-review by the editor, the submitted manuscripts are first assessed by a biostatistics reviewer and are then forwarded to reviewers of the related discipline if the biostatistics reviewers approve the study; on the other hand, some other journals ask the reviewers of the related disciplines to assess and forward the manuscripts to the biostatistics reviewer if appropriate. Submitting the manuscripts first to the biostatistics reviewer rather than the reviewers of the related discipline would save time. Even when reviewers of the related discipline accept the submitted manuscripts, errors can be found by the biostatistics reviewer in the analysis and presentation of the data, which require the revision of a manuscript. This may result in alterations in the interpretation of the data and the writing of the discussion, which ultimately causes the reviewers of the related discipline to review the manuscript again, which leads to unnecessary delays in the review process. ${ }^{3}$

Reviewers are expected to take part in the task of prevention of statistical errors in publications. The reviewer of a manuscript, who is an expert in the subject matter areas related to the evaluation, should request from the editor that the manuscript be reviewed by a biostatistics specialist. From researchers' point of view, the following main issues should be considered to prevent the occurrence of statistical errors during the process of a scientific study and in the phase of publishing,

1) Understanding the role and importance of the science of biostatistics in scientific research

2) Knowledge of basic biostatistics

3) Consultation with a biostatistics specialist in the planning, analysis and interpretation stages of the research

4) Review by a biostatistics specialist before submitting the manuscript to a journal

5) The statistical approach from other similar research studies should not be copied

6) Avoiding overreliance and overconfidence on "user friendly" software programs just by ease they seem to provide, being cautious and distrustful

7) When writing the manuscript, avoiding a "copy-paste" approach from the text of other publications.

\section{Conclusion}

All in all, the most important factor in preventing statistical 
errors in publications is having the editor submit all manuscripts for a statistical review. Nothing can be done for errors that cannot be detected in the process of publication; nevertheless, prospectively speaking, it is important that the journal editors publish papers that carry educational qualifications on statistical topics, and are oriented towards statistical errors committed in research studies, in the sense of informing the researchers and drawing attention to the topic.

\section{Acknowledgement}

None.

\section{Conflict of Interest}

None.

\section{Refereces}

1. Ercan I, Yazici B, Yang Y, et al. Misusage of statistics in medical research Eur J Gen Med. 2007;4(3):128-134.

2. McGuigan S. The use of statistics in the British Journal of Psychiatry. $\mathrm{Br}$ J Psychiatry. 1995;167(5):683-688.

3. Ercan I, Ocakoglu G, Sigirli D, et al. Assessment of Submitted Manuscripts in Medical Sciences According to Statistical Errors. Turkiye Klinikleri J Med Sci. 2012;32(5):1381-1387.
4. He J, Jin Z, Yu D. Statistical reporting in Chinese biomedical journals. Lancet. 2009;373(9681):2091-2093.

5. Wang Q, Zhang B. Research design and statistical methods in Chinese medical journals. JAMA. 1998;280(3):283-285.

6. Gardner MJ, Bond J. An exploratory study of statistical assessment of papers published in the British Medical Journal. JAMA. 1990;263(10):1355-1357.

7. Altman DG. The scandal of poor medical research. BMJ. 1994;308(6924):283-284.

8. George SL. Statistics in medical journals: a survey of current policies and proposal for editors. Pediatr Blood Cancer. 1985;13(2):109-112.

9. Goodman SN, Altman DG, George SL. Statistical reviewing policies of Medical Journals Caveat Lector? J Gen Intern Med. 1998;13(11):753756.

10. MacArthur RD, Jackson GG. An evaluation of the use of statistical methodology in the Journal of Infectious Diseases. J Infect Dis. 1984;149(3):349-354.

11. Strasak AM, Zaman Q, Pfeiffer KP, et al. Statistical errors in medical research - a review of common pitfalls. Swiss Med Wkly. 2007;137(34):44-49. 